\title{
A four-year cardiovascular risk score for type 2 diabetic inpatients
}

Dolores Ramírez-Prado, Antonio Palazón-Bru, David M Folgado-de la Rosa, María Á Carbonell-Torregrosa, Ana M Martínez-Díaz, Damian RJ Martínez-St. John, Vicente F Gil-Guillén

As cardiovascular risk tables currently in use were constructed using data from the general population, the cardiovascular risk of patients admitted via the hospital emergency department may be underestimated. Accordingly, we constructed a predictive model for the appearance of cardiovascular diseases in patients with type 2 diabetes admitted via the emergency department. We undertook a four-year follow-up of a cohort of 112 adult patients with type 2 diabetes admitted via the emergency department for any cause except patients admitted with acute myocardial infarction, stroke, cancer, or a palliative status. The sample was selected randomly between 2010 and 2012. The primary outcome was time to cardiovascular disease. Other variables (at baseline) were gender, age, heart failure, renal failure, depression, asthma/chronic obstructive pulmonary disease, hypertension, dyslipidaemia, insulin, smoking, admission for cardiovascular causes, pills per day, walking habit, fasting blood glucose and creatinine. A cardiovascular risk table was constructed based on the score to estimate the likelihood of cardiovascular disease. Risk groups were established and the c-statistic was calculated. Over a mean follow-up of 2.31 years, 39 patients had cardiovascular disease (34.8\%, 95\% Cl: 26.0-43.6\%).

Predictive factors were gender, age, hypertension, renal failure, insulin, admission due to cardiovascular reasons and walking habit. The c-statistic was 0.734 (standard error: 0.049). After validation, this study will provide a tool for the primary health care services to enable the short-term prediction of cardiovascular disease after hospital discharge in patients with type 2 diabetes admitted via the emergency department. 
2 AUTHORS: Dolores Ramírez-Prado ${ }^{1,2}$, Antonio Palazón-Bru ${ }^{1,2}$, David Manuel Folgado-de la Rosa ${ }^{2}$,

3 María Ángeles Carbonell-Torregrosa ${ }^{3}$, Ana María Martínez-Díaz ${ }^{3}$, Damian Robert James Martínez-St.

$4 \mathrm{John}^{2}$, Vicente Francisco Gil-Guillén ${ }^{1,2}$.

5 Institutions:

$6 \quad{ }^{1}$ Research Unit, Elda Hospital, Elda, Alicante, Spain.

7 2Department of Clinical Medicine, Miguel Hernández University, San Juan de Alicante, Alicante,

8 Spain.

$9 \quad{ }^{3}$ Emergencies Unit, Elda Hospital, Elda, Alicante, Spain.

10 Corresponding author: Antonio Palazón-Bru, Department of Clinical Medicine, Miguel Hernández

11 University, San Juan de Alicante, Spain. E-mail: antonio.pb23@gmail.com. Phone: +34 965919449.

12 Fax: +34 965919450. 
INTRODUCTION

14 Cardiovascular diseases (CVD) constitute one of the main causes of death worldwide, and one of the

15 main reasons for admission via the hospital emergency department (ED) (Fan et al., 2011; WHO,

16 2014). The most important risk factors for CVD include diabetes mellitus, hypertension, dyslipidaemia,

17 obesity and smoking (WHO, 2007). These factors are all prevalent among patients admitted via the ED

18 (Cinza Sanjurjo et al., 2006; Fan et al., 2011).

One in every six ED admissions among diabetic patients is related with the diabetes itself, with

almost half of these admissions due to glycaemic decompensation. The other main reasons (unrelated with the diabetes) for ED admissions among these patients are lesions and poisonings (Hinojosa MenaBernal et al., 2004).

We are unaware of any studies in patients with type 2 diabetes admitted via the ED that have analyzed the onset of CVD and constructed a predictive model to indicate which of these patients have a greater likelihood of presenting CVD. Although there exist cardiovascular risk tables constructed with data from the general population, health centres, working persons and volunteers, the results of these tables are not based on the follow-up of patients with specific disorders, such as type 2 diabetes (Cooney, Dudina \& Graham, 2009). Thus, the cardiovascular risk obtained from these tables might be under-estimated, as we must consider that diabetic persons admitted via the ED present important differences (highly heterogenic) with the type of patients used for the construction of these scales and tables. For example, diabetic patients admitted via the ED have, a priori, more disorders. Accordingly, we undertook a study with a four-year follow-up at the Elda Hospital (Spain) to construct a predictive model of CVD. Once validated (by reproducing our results in other populations) and after hospital discharge, this model could be used preventively by the primary health care services with the aim of reducing the cardiovascular mortality and morbidity in patients with type 2 diabetes admitted via the ED. 
MATERIALS \& METHODS

39 Study population, design and participants, ethical considerations

40 The study population was formed by diabetic patients admitted via the ED in the Valle de Elda

41 healthcare area (Valencian Community), an industrial area with 198,090 inhabitants with a low-to-

42 medium socioeconomic level (Martínez-Orozco et al. 2015). The ED of Elda Hospital (a public

43 institution) attends about 160 general emergency cases daily among the adult population, not including

44 obstetric and gynaecologic cases (Carbonell Torregrosa et al. 2014).

The study cohort comprised type 2 diabetic patients admitted for any reason via the ED of Elda

46

47

48

49

50

51

52

Hospital (only hospital in the healthcare area), aged $>13$ years (patients younger than 13 years are seen by the paediatric services), who were willing to participate. The follow-up was four years. Patients were excluded if they were pregnant or had a personal history of acute myocardial infarction, stroke, or cancer, or were receiving palliative care. A random sample was selected from all patients admitted via the ED between January 2010 and March 2012. The sampling procedure involved random selection of one day every week and recruiting all the diabetic patients who fulfilled the criteria and were admitted on that day.

Patients with type 1 diabetes were not included in this study because they generally have different characteristics to patients with type 2 diabetes; for example, patients with type 1 diabetes are usually younger. Grouping together two non-homogenous groups of patients would produce results that would not really be useful in daily clinical practice. For this reason, most authors usually analyze different outcomes depending on the type of diabetes (Ramírez-Prado et al., 2015). Each patient was followed from the recruitment date until he or she had a CVD, whether fatal or not. If no CVD developed, the patient was followed for four years (if still alive), or until the date of last clinical contact (assuming the patient had had no CVD by this date). 
The study posed no additional risk to the patients and an indirect benefit was expected, as the results might reduce short-term cardiovascular morbidity and mortality in this type of patient. The study was carried out in compliance with the principles of the World Medical Association Declaration of Helsinki and complied with the European Union norms of good clinical practice. The patients were informed verbally about the study and about the information required. The study was approved by the Ethics Committee of the Elda Department of Health (Ref. UI13016).

\section{Variables and measurements}

The main outcome variable was cardiovascular morbidity or mortality during the four-year follow-up.

Cardiovascular conditions were considered to be those affecting the heart or blood vessels

(cerebrovascular, legs, kidneys or heart) (Bonny et al., 2008).

Data collected at admission (baseline) included gender, age (years), personal history of diseases (heart failure, renal failure, depression, asthma/chronic obstructive pulmonary disease (COPD), hypertension and dyslipidaemia), use of insulin, smoking, admission due to cardiovascular reasons, number of tablets per day (usual medication for whatever condition, excluding diabetes therapy), walking habit, fasting blood glucose $(\mathrm{mmol} / \mathrm{L})$ and creatinine $(\mu \mathrm{mol} / \mathrm{L})$.

Cardiovascular morbidity and mortality was assessed during the four-year follow-up by regularly checking the hospital and health centre records. In the case of any doubt about death, contact was made with the patient (if alive) or that patient's relatives, or by contacting the patient's assigned physician (if there was still doubt). Information about gender, age, personal history of diseases, smoking, taking of insulin, and number of pills daily was obtained by patient interview and corroborated from the medical records. Information about walking habits was obtained just at the interview. Data regarding admissions were obtained from the hospital records. The baseline fasting 
83 blood glucose and the creatinine were measured according to the current clinical guidelines (American

84 Diabetes Association, 2014).

85 Sample size and statistical methods

86 The final cohort sample was 112 patients. Assuming 95\% confidence, an expected censored proportion

87 of $60 \%$, an exposure proportion of $35 \%$ and an expected hazard ratio (HR) of 2.50 , the power to

88 contrast a HR different to 1 was calculated. The resulting value, obtained from implementing the

89 formula for the power in an Excel spreadsheet and solving it with the Solver tool, was 83.28\%.

90

91

92

93

As smoking and walking had lost values, $32.4 \%$ and $24.1 \%$ respectively, 100 multiple

imputations were made beforehand using logistic regression switching with predictive mean matching.

This is the most suitable procedure when the number of missing data is between $10-50 \%$. In this way we were able to work with all the variables (Marshall, Altman \& Holder, 2010).

Absolute and relative frequencies were used to describe the qualitative variables, with means and standard deviations for the quantitative variables. A Cox multivariate regression model was constructed to determine which variables were associated with cardiovascular morbidity and mortality, calculating the HR. As we had few patients, we selected a maximum number of explanatory variables in the model. As a heuristic rule we considered there needed to be at least 10 observations of morbidity and mortality or no morbidity and mortality for each explanatory variable. To obtain the variables in the model we analyzed all the possible combinations with a maximum of 7 variables $(16,383)$, calculating the value of the c-statistic in all of them. The combination with the highest value was then selected. The c-statistic is similar to the area under the ROC curve, but the former takes into account censoring. The goodness of fit of the model was assessed by the score (log-rank) test. Using the $\beta$ coefficients of the multivariate model a risk table was constructed based on the sum of the points to estimate the likelihood of CVD (Sullivan, Massaro \& D'Agostino, 2004). After calculating the scores and their associated risk, risk groups were designed: low risk ( $<5$ th percentile), medium risk (from the 
107 5th percentile to the median), high risk (from the median to the 95th percentile), and very high risk

108 ( $\geq 95$ th percentile). All the analyses were done with an $\alpha=5 \%$ and for each relevant parameter the

109 associated confidence interval (CI) was calculated. All the analyses were done with IBM SPSS

110 Statistics 19 and R 2.13.2.

111

112 RESULTS

113 Of a total of 115 patients who fulfilled the inclusion criteria, three were excluded because there was no

114 further contact after the initial visit (lost during the follow-up). Thus, the final sample comprised 112 115 patients.

116 Over a mean follow-up of $2.3 \pm 1.6$ years, 39 of the 112 patients had CVD $(34.8 \%, 95 \%$ CI:

$11726.0-43.6 \%)$. Of these, 22 were fatal (19.6\%, 95\% CI: 12.3-27.0\%) (cardiac arrest, 12; ischaemic heart

118 disease, 3 ; heart failure, 3 ; stroke, 3 ; peripheral arterial disease, 1$)$ and 17 were non fatal $(15.2 \%, 95 \%$

119 CI: 8.5-21.8\%) (ischaemic heart disease, 7; heart failure, 6; atrial fibrillation, 2; renal failure, 1;

120 pericarditis, 1) (Table 1). This represents an incidence density of 150 CVD for each 1000 person-years

121 (95\% CI: 107-206 CVD per 1000 person-years), of which 104 were fatal (95\% CI: 69-152 CVD per

1221000 person-years) and 46 non fatal (95\% CI: 89-273 CVD per 1000 person-years).

123

124 was advanced (70.5 years); the youngest patient was 34 years old. There was a high prevalence of

125 comorbidity (heart failure, 13.4\%; renal failure, 8.9\%; depression, 8.9\%; asthma/COPD, 13.4\%;

126 hypertension, 75.0\%; dyslipidaemia, 42.9\%) and a very high mean number of daily pills (5.6).

127 Concerning lifestyle habits, $21.4 \%$ of the patients smoked and $26.8 \%$ walked usually. For the diabetes-

128 related variables, $43.8 \%$ used insulin and the mean baseline fasting blood glucose was $8.4 \mathrm{mmol} / \mathrm{L}$.

129 Notably, $26.8 \%$ of the patients were admitted with a cardiovascular problem. 
$1310.90-3.75, \mathrm{p}=0.095)$, older age (per 1 year $)(\mathrm{HR}=1.04,95 \% \mathrm{CI}: 1.00-1.08, \mathrm{p}=0.031)$, hypertension

$132(\mathrm{HR}=1.11,95 \% \mathrm{CI}: 0.47-2.62, \mathrm{p}=0.804)$, renal failure $(\mathrm{HR}=2.76,95 \% \mathrm{CI}: 1.01-7.59, \mathrm{p}=0.048)$, insulin 133 use $(\mathrm{HR}=1.56,95 \% \mathrm{CI}: 0.77-3.16, \mathrm{p}=0.212)$, admission for cardiovascular reasons $(\mathrm{HR}=2.15,95 \% \mathrm{CI}$ :

$1341.09-4.25, \mathrm{p}=0.027)$ and not having the habit of walking $(\mathrm{HR}=0.57,95 \% \mathrm{CI}: 0.25-1.31, \mathrm{p}=0.185)$. The 135 model obtained with these factors was very significant $(\mathrm{p}<0.001)$. The scores for each variable in the 136 predictive model and the risk groups are shown in Figure 1. The c-statistic for the scoring system was 1370.734 (standard error: 0.049).

Figure 2 shows that there were significant differences in survival between the various risk 139 groups $(\mathrm{p}<0.001)$, with a reduction in survival as the risk category increased.

\section{DISCUSSION}

142 Summary

143 This study constructed a predictive model for CVD with a good discriminating power

144 (c-statistic $=0.734)$ indicating which patients with type 2 diabetes who are admitted via the ED have a 145 greater risk of presenting CVD, either fatal or non fatal.

\section{Strengths and limitations}

147 The main strength of this study is related to the lack of other studies that have constructed short-term 148 predictive models for CVD in patients with type 2 diabetes admitted via the ED. The innovative results 149 can therefore be used to help take decisions to try to avoid the onset of CVD. Additionally, the 150 predictive model constructed had a good discriminating power (c-statistic $=0.734$ ), which will enable 151 precise predictions after validation. 
154 discriminating power of the scale constructed (Cooney, Dudina \& Graham, 2009). We were therefore

155 very rigorous designing the model, selecting a maximum number of variables with a stepwise

156 procedure. The results obtained in the model indicate a high degree of significance ( $p<0.001$ for the

157 goodness of fit), accompanied by a c-statistic above $70 \%(0.734)$. Furthermore, the contrast power in

158 our sample size calculation was $83.28 \%$.

159 To minimize the possible bias related with measurement and selection, calibrated devices were

160 used and a random sample was selected. However, we were unable to use certain variables that are

161 important in the development of CVD, e.g., obesity, years with diabetes, HbA1c, because the

162 emergency department protocol in our hospital does not include their measurement. If they had been

163 taken into account, then the c-statistic may well have improved. Nevertheless, the resulting value

164 without the inclusion of these variables was satisfactory. Finally, part of the values related to the

165 variable walking habit was obtained by statistical imputation, though the procedure used is considered

166 adequate for this type of model (Marshall, Altman \& Holder, 2010).

167 Comparison with existing literature

168 Others have constructed cardiovascular risk models that have been extensively validated. However,

169 these models were based on the general population, or patients attending their healthcare centre,

170 working persons or volunteers. Our patients, though, formed a heterogeneous group concerning

171 prognostic factors for CVD among the populations used to construct the existing models. A priori, they

172 were all less stable and all had type 2 diabetes. These differences make comparison with current

173 cardiovascular risk tables very difficult. Nonetheless, the c-statistic for internal validation $(0.734)$ is

174 within the range obtained by the other cardiovascular models $(0.708-0.82)$. This indicates that, if our

175 model is validated externally with results similar to the internal validation, it could be used in daily

176 clinical practice (Cooney, Dudina \& Graham, 2009). 
The prognostic factors for CVD in our study were: insulin, older age, male gender, renal failure,

178 hypertension, habit of walking, and being admitted for cardiovascular reasons. These results

179 corroborate those of other authors, except for the initial admission due to cardiovascular problems

180 (Muggeo et al., 2000; Bo et al., 2005; Hong Kong Diabetes Registry et al., 2008; Kleefstra et al., 2008;

181 Cooney, Dudina \& Graham, 2009), although this association was very logical. Finally, smoking was

182 notably absent in the predictive model, possibly due to the already high underlying cardiovascular risk

183 of these patients (Gil-Guillén et al., 2009).

\section{Implications for research and/or practice}

185 After validation, this study could provide clinical practice with a tool to predict premature

186 cardiovascular morbidity and mortality in patients with type 2 diabetes admitted via the ED. If our

187 results are confirmed with other studies, those patients who have a high likelihood of CVD within four

188 years should be closely followed with effect from their hospital discharge. The control should be based

189 mainly on medication adjustment, control of therapeutic non-compliance, and ensuring a healthy

190 lifestyle (Ramírez-Prado et al., 2015).

191 This validation will require recruiting a new sample of patients and determining the two key

192 questions with this sample; firstly, whether the scoring system correctly discriminates between those

193 patients who have CVD and those who do not (using the c-statistic), and secondly, whether the

194 proportion of observed events is similar to that given by the model (using $\mathrm{X}^{2}$ tests). This validation is

195 currently the subject of study in our hospital, and obviously it could also be done in other geographical

196 areas, such that if the two previous conditions are verified, a tool will be available to help reduce the

197 incidence of CVD in patients with similar characteristics to those of the present study sample.

199 CONCLUSIONS 
200 This study provides a tool that, after validation, will enable short-term cardiovascular morbidity and 201 mortality to be predicted in patients with type 2 diabetes admitted via the ED. This tool should be used

202 by the primary health care services to improve the prognosis, by making more suitable decisions and 203 planning the beneficial needs of the patient, though whenever possible indicating that the patient 204 should walk and carrying out stricter control in those patients who present a high cardiovascular risk. 205

206 ACKNOWLEDGMENTS

207 We thank all the services of the General Hospital of Elda who participated in this study. The authors 208 also thank Ian Johnstone for help with the English language version of the text.

209

210

211

212 
REFERENCES

214 American Diabetes Association. 2014. Standards of medical care in diabetes--2014. Diabetes Care 37

215 Suppl 1:S14-80.

216

217 Bo S, Ciccone G, Rosato R, Gancia R, Grassi G, Merletti F, Pagano GF. 2005. Renal damage in 218 patients with Type 2 diabetes: a strong predictor of mortality. Diabetic Medicine 22:258-265.

220 Bonny A, Lacombe F, Yitemben M, Discazeaux B, Donetti J, Fahri P, Megbemado R, Estampes B. 221 2008. The $2007 \mathrm{ESH} / \mathrm{ESC}$ guidelines for the management of arterial hypertension. Journal of 222 Hypertension 26:825; author reply 825-6.

224 Carbonell Torregrosa MA, Urtubia Palacios A, Palazón Bru A, Carrasco Tortosa V, Gil Guillén V. 225 2014. Impacto de la implantación del programa ASIGNA en un servicio de urgencias hospitalario.

226 Emergencias 26:188-194.

228 Cinza Sanjurjo S, Cabarcos Ortiz de Barrón A, Nieto Pol E, Torre Carballada JA. 2006. Prevalencia de 229 hipertensión arterial en población mayor de 65 años ingresada en un Servicio de Medicina Interna. 230 Anales de Medicina Interna 23:577-581.

232 Cooney MT, Dudina AL, Graham IM. 2009. Value and limitations of existing scores for the 233 assessment of cardiovascular risk: a review for clinicians. Journal of the American College of 234 Cardiology 54:1209-1227. 
236 Fan L, Shah MN, Veazie PJ, Friedman B. 2011. Factors associated with emergency department use 237 among the rural elderly. Journal of Rural Health 27:39-49.

Gil-Guillén VF, Merino-Sánchez J, Sánchez-Ruiz T, Amorós-Barber T, Aznar-Vicente J, Abellán-

Alemán J, Llisterri-Caro JL, Orozco-Beltrán D, Pascual Pérez M, Márquez Contreras E. 2009.

Valoración del riesgo cardiovascular en la fase longitudinal del estudio Mediterránea. Revista Clínica

Española 209:118-30.

244 Hinojosa Mena-Bernal MC, González Sarmiento E, Hinojosa Mena-Bernal J, Zurro Hernández J. 2004.

245 Asistencia urgente del paciente diabético en el área este de la provincia de Valladolid. Anales de 246 Medicina Interna 21:7-11.

248 Hong Kong Diabetes Registry, Yang X, So WY, Tong PC, Ma RC, Kong AP, Lam CW, Ho CS, 249 Cockram CS, Ko GT, Chow CC, Wong VC, Chan JC. 2008. Development and validation of an all250 cause mortality risk score in type 2 diabetes. Archives of Internal Medicine 168:451-457.

252 Kleefstra N, Landman GW, Houweling ST, Ubink-Veltmaat LJ, Logtenberg SJ, Meyboom-de Jong B, 253 Coyne JC, Groenier KH, Bilo HJ. 2008. Prediction of mortality in type 2 diabetes from health-related 254 quality of life (ZODIAC-4). Diabetes Care 31:932-933.

256 Marshall A, Altman DG, Holder RL. 2010. Comparison of imputation methods for handling missing 257 covariate data when fitting a Cox proportional hazards model: a resampling study. BMC Medical 258 Research Methodology 10:112. 
260 Martínez-Orozco MJ, Perseguer-Torregrosa Z, Gil-Guillén VF, Palazón-Bru A, Orozco-Beltran D,

261 Carratalá-Munuera C. 2015. Suitability of antiplatelet therapy in hypertensive patients. Journal of 262 human hypertension 29:40-45.

263

264 Muggeo M, Zoppini G, Bonora E, Brun E, Bonadonna RC, Moghetti P, Verlato G. 2000. Fasting 265 plasma glucose variability predicts 10-year survival of type 2 diabetic patients: the Verona Diabetes 266 Study. Diabetes Care 23:45-50.

267

268 Ramírez-Prado D, Palazón-Bru A, Folgado-de-la Rosa DM, Carbonell-Torregrosa MA, Martínez-Díaz 269 AM, Gil-Guillén VF. 2015. Predictive models for all-cause and cardiovascular mortality in type 2 270 diabetic inpatients. A cohort study. International Journal of Clinical Practice 69:474-484.

272 Sullivan LM, Massaro JM, D'Agostino RB Sr. 2004. Presentation of multivariate data for clinical use:

273 The Framingham Study risk score functions. Statistics in Medicine 23:1631-1660. Review.

275 World Health Organization. 2007. Prevention of cardiovascular disease. Available at

276 http://whqlibdoc.who.int/publications/2007/9789241547178 eng.pdf?ua=1 (accessed May 2014).

278 World Health Organization. 2014. The top 10 causes of death. Available at

279 http://www.who.int/mediacentre/factsheets/fs310/en/ (accessed January 2015). 


\section{1}

Four-year risk score for predicting cardiovascular disease in type 2 diabetic inpatients.

\begin{tabular}{cc}
\hline Insulin & Score \\
\hline Yes & 2 \\
No & 0 \\
\hline
\end{tabular}

\begin{tabular}{cc}
\hline Hypertension & Score \\
\hline Yes & 1 \\
No & 0 \\
\hline
\end{tabular}

\begin{tabular}{cc}
\hline Age (years) & Score \\
\hline$<40$ & 0 \\
$40-49$ & 2 \\
$50-59$ & 4 \\
$60-69$ & 6 \\
$70-79$ & 8 \\
$\geq 80$ & 10 \\
\hline
\end{tabular}

\begin{tabular}{cc}
\hline $\begin{array}{c}\text { Personal } \\
\text { history of renal } \\
\text { failure }\end{array}$ & Score \\
\hline Yes & 5 \\
\hline No & 0 \\
\hline
\end{tabular}

\begin{tabular}{cc}
\hline $\begin{array}{c}\text { Admitted due to } \\
\text { cardiovascular reasons }\end{array}$ & Score \\
\hline Yes & 4 \\
No & 0 \\
\hline
\end{tabular}

\begin{tabular}{cc}
\hline Gender & Score \\
\hline Male & 3 \\
Female & 0 \\
\hline
\end{tabular}

\begin{tabular}{cc}
\hline $\begin{array}{c}\text { Habit of } \\
\text { walking }\end{array}$ & Score \\
\hline Yes & -3 \\
\hline No & 0 \\
\hline
\end{tabular}

Cardiovascular risk (\%)

\begin{tabular}{ccccc}
\hline Total score & 1 year & 2 years & 3 years & 4 years \\
\hline Low (<5) & $<8.00$ & $<8.35$ & $<10.85$ & $<13.85$ \\
Medium (5-11) & $9.65-28.95$ & $10.10-30.10$ & $13.05-37.55$ & $16.65-45.75$ \\
High (12-18) & $34.10-75.45$ & $35.45-77.05$ & $43.75-85.55$ & $52.65-91.90$ \\
Very high ( $\geq 19)$ & $>82.00$ & $>83.45$ & $>90.55$ & $>95.35$ \\
\hline
\end{tabular}


2

Survival of the different risk groups for cardiovascular disease of type 2 diabetic inpatients in a Spanish region.

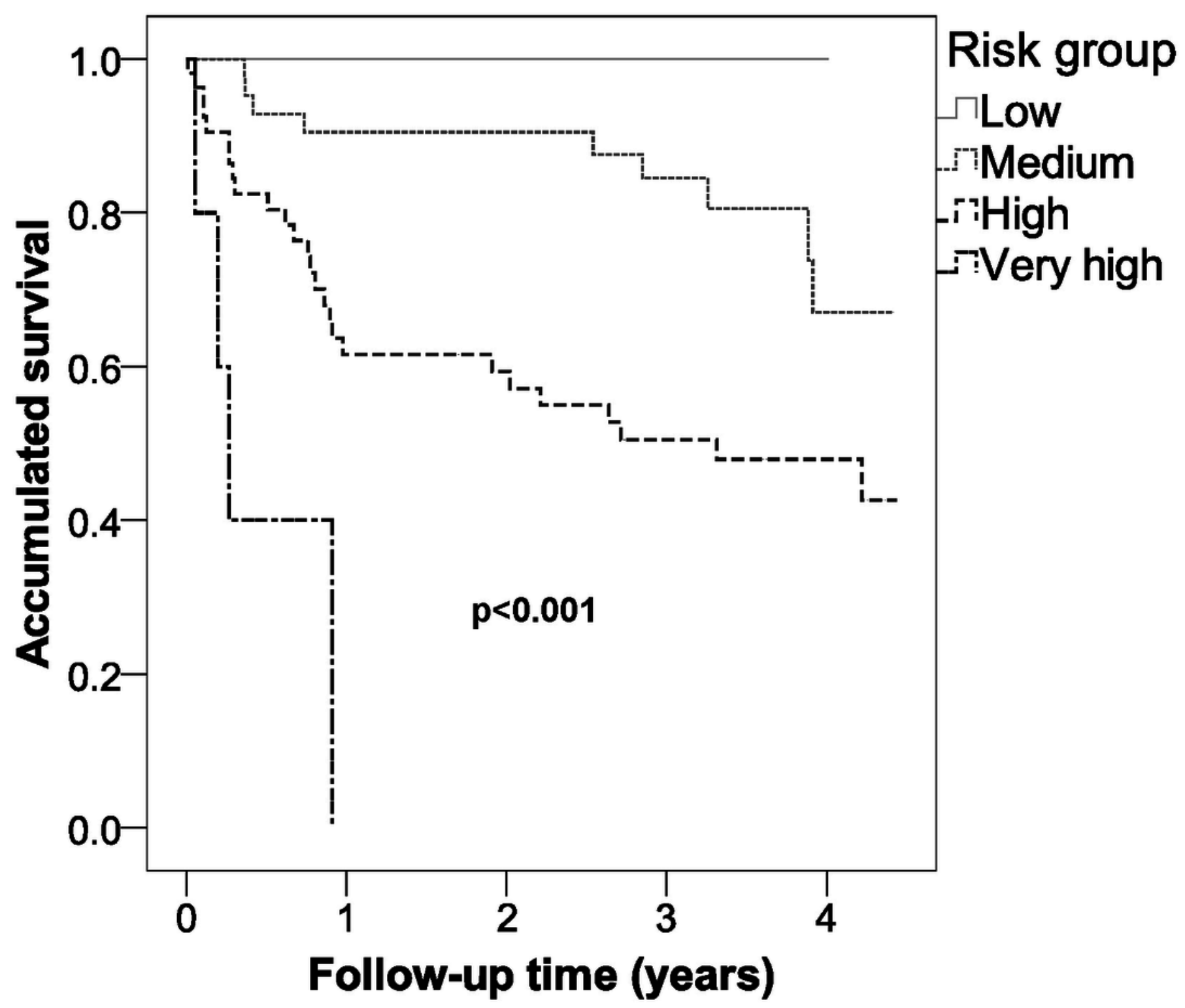




\section{Table $\mathbf{1}_{\text {(on next page) }}$}

Baseline characteristics and adjusted hazard ratios for cardiovascular disease for type 2 diabetic inpatients in a Spanish region. 2010-2012 data.

Abbreviations: $\mathrm{HR}$, hazard ratio; $\mathrm{Cl}$, confidence interval; $\mathrm{COPD}$, chronic obstructive pulmonary disease; FBG, fasting blood glucose; N/A, not applicable; N/M, not in the model. Goodness-offit of the model: $X^{2}=24.43, p<0.001$. c-statistic: 0.734 (standard error: 0.049 ). 


\begin{tabular}{|c|c|c|c|c|}
\hline Variable & $\begin{array}{c}\text { Total }(n=112) \\
n(\%) / x \pm s\end{array}$ & HR & $95 \% \mathrm{CI}$ & p-value \\
\hline \multicolumn{5}{|l|}{ Cardiovascular morbidity: } \\
\hline Ischemic heart disease & $7(6.2)$ & \multirow{5}{*}{ N/A } & \multirow{5}{*}{$\mathrm{N} / \mathrm{A}$} & \multirow{5}{*}{$\mathrm{N} / \mathrm{A}$} \\
\hline Heart failure & $6(5.4)$ & & & \\
\hline Atrial fibrillation & $2(1.8)$ & & & \\
\hline Renal failure & $1(0.9)$ & & & \\
\hline Pericarditis & $1(0.9)$ & & & \\
\hline \multicolumn{2}{|l|}{ Cardiovascular mortality: } & \multirow{6}{*}{$\mathrm{N} / \mathrm{A}$} & \multirow{6}{*}{$\mathrm{N} / \mathrm{A}$} & \multirow{6}{*}{$\mathrm{N} / \mathrm{A}$} \\
\hline Cardiac arrest & $12(10.7)$ & & & \\
\hline Ischemic heart disease & $3(2.7)$ & & & \\
\hline Heart failure & $3(2.7)$ & & & \\
\hline Cerebral haemorrhage & $3(2.7)$ & & & \\
\hline Peripheral arterial disease & $1(0.9)$ & & & \\
\hline Male gender & $59(52.7)$ & 1.84 & $0.90-3.75$ & 0.095 \\
\hline Age (years) & $70.5 \pm 12.4$ & 1.04 & $1.00-1.08$ & 0.031 \\
\hline Depression & $10(8.9)$ & $\mathrm{N} / \mathrm{M}$ & $\mathrm{N} / \mathrm{M}$ & $\mathrm{N} / \mathrm{M}$ \\
\hline Asthma/COPD & $15(13.4)$ & $\mathrm{N} / \mathrm{M}$ & $\mathrm{N} / \mathrm{M}$ & $\mathrm{N} / \mathrm{M}$ \\
\hline Hypertension & $84(75.0)$ & 1.11 & $0.47-2.62$ & 0.804 \\
\hline Dyslipidaemia & $48(42.9)$ & $\mathrm{N} / \mathrm{M}$ & $\mathrm{N} / \mathrm{M}$ & $\mathrm{N} / \mathrm{M}$ \\
\hline Heart failure & $15(13.4)$ & $\mathrm{N} / \mathrm{M}$ & $\mathrm{N} / \mathrm{M}$ & $\mathrm{N} / \mathrm{M}$ \\
\hline Renal failure & $10(8.9)$ & 2.76 & $1.01-7.59$ & 0.048 \\
\hline Insulin & $49(43.8)$ & 1.56 & $0.77-3.16$ & 0.212 \\
\hline Smoking & $24(21.4)$ & $\mathrm{N} / \mathrm{M}$ & $\mathrm{N} / \mathrm{M}$ & $\mathrm{N} / \mathrm{M}$ \\
\hline Admission for cardiovascular reasons & $30(26.8)$ & 2.15 & $1.09-4.25$ & 0.027 \\
\hline Pills per day & $5.6 \pm 3.9$ & $\mathrm{~N} / \mathrm{M}$ & $\mathrm{N} / \mathrm{M}$ & $\mathrm{N} / \mathrm{M}$ \\
\hline Habit of walking & $30(26.8)$ & 0.57 & $0.25-1.31$ & 0.185 \\
\hline FBG (mmol/L) & $8.4 \pm 4.4$ & $\mathrm{~N} / \mathrm{M}$ & $\mathrm{N} / \mathrm{M}$ & $\mathrm{N} / \mathrm{M}$ \\
\hline Creatinine $(\mu \mathrm{mol} / \mathrm{L})$ & $97.2 \pm 44.2$ & $\mathrm{~N} / \mathrm{M}$ & $\mathrm{N} / \mathrm{M}$ & $\mathrm{N} / \mathrm{M}$ \\
\hline
\end{tabular}

